\title{
Amelioration of Diabetic Nephropathy in Streptozotocin-Induced Diabetic Rats by Acacia catechu Leaves Extract
}

\author{
Prima D’souza' Rajendra Holla ${ }^{2}$ Gangadhara Swamy ${ }^{3}$ \\ ${ }^{1}$ Department of Anatomy, K. S. Hegde Medical Academy, \\ Deralakatte, Mangalore, Karnataka, India \\ 2Department of Pharmacology, K. S. Hegde Medical Academy, \\ Deralakatte, Mangalore, Karnataka, India \\ Address for correspondence Gangadhara Swamy, MSc, \\ Department of Anatomy, Subbaiah Institute of Medical Sciences and \\ Research Center, Shivamogga, Karnataka, 577222, India \\ (e-mail: dr.gangadharaswamy@gmail.com).
}

${ }^{3}$ Department of Anatomy, Subbaiah Institute of Medical Sciences and Research Center, Shivamogga, Karnataka, India

J Health Allied Sci Nu 2019;9:116-120

\begin{abstract}
Objective The present study was performed to evaluate the ethanolic extract of leaves of Acacia catechu (A. catechu) for its effect on streptozotocin (STZ)-induced diabetes mellitus (DM) and its renal complications in male Wistar albino rats.

Materials and Methods Male Wistar albino rats were grouped into control (A), STZ-induced DM (B), STZ-induced DM rats with A. catechu orally of $75 \mathrm{mg} / \mathrm{kg}$ body weight (kbw) for 35 days $(C)$, with each group having six rats $(n=6)$ weighing between 200 to $250 \mathrm{~g}$ each. Group A receives only water, orally; group B receives a single dose of STZ at $45 \mathrm{mg} / \mathrm{kbw}$ intraperitoneal administration (IP); group C receives STZ IP and oral A. catechu for 35 days. On the 36th day, animals were euthanized, the kidney tissues were analyzed for biochemical parameters, such as GOT (glutamic oxaloacetic transaminase), GPT (glutamic pyruvic transaminase), oxidative stress assessment parameters, and histopathological studies.

Results In group C rats, activities of the enzymes were nearer to group A when

Keywords

- diabetes mellitus

- Streptozotocin

- Acacia catechu

- nephroprotective compared with group B. Histopathological findings were also suggesting that renal toxicity were observed at a lesser extent in group $C$.

Conclusion The ethanolic extract of $A$. catechu signified as nephroprotective effect. The present data could provide adequate confirmation of the efficacy of ethanolic extract of leaves of $A$. catechu for further experimental studies on a standardized formulation.
\end{abstract}

\section{Introduction}

Diabetes mellitus (DM) is a metabolic disorder characterized by hyperglycemia, glycosuria, and negative nitrogen balance and it is mainly due to absolute deficiency or diminished effectiveness of insulin. Survey reports had suggested that currently, 366 million people are diabetic in the world and also been predicted that it will reach up to 552 million people by $2030 .{ }^{1}$ With the current antidiabetes therapies, it cannot be completely curable. The mortality in DM is accounted for its complications, such as nephropathy, neuropathy, and retinopathy. It is estimated that approximately world's $30 \%$ of diabetic patients progress to diabetic nephropathy (DNP). The high concentrations of blood sugar damages the kidney tissues thus leading to altered kidney function in the patients, causing DNP and develop an end-stage renal disease. ${ }^{2}$ DM is an important etiopathological factor in oxidative stress. ${ }^{3}$ As a result of lipid and protein oxidation, the levels of superoxide dismutase (SOD), glutathione peroxidase (GSH-Px) increases in kidneys. ${ }^{4-6}$ Previous studies have demonstrated that DM exhibits enhanced oxidative stress and highly reactive oxygen species (ROS) in pancreatic islets due to persistent and chronic hyperglycemia, thereby depletes the activity of the received

September 26, 2019 accepted after revision

November 18, 2019
License terms

()(1) $\ominus \circledast$ 
antioxidative defense system, and thus promotes the free radical generation. ${ }^{7}$

In DM patients, there is an increase in the level of type-IV collagen fibers with the concomitant decrease in the level of laminin and heparan sulfate, thus affecting the pore size and selectivity, causing kidney damage. The kidney damage in DNP is manifested histologically by the thickening of the glomerular basement membrane, mesangial matrix expansion, macrophage infiltration, podocyte loss, and tubular epithelial degeneration. ${ }^{8}$ Existing therapy for DM is known to support glycemic control but it is believed to do little in regard to the complications to the various organs. Besides, these antidiabetic drugs are associated with mild-to-moderate sideeffects. ${ }^{9}$

Though different types of oral hypoglycemic agents are available along with the insulin for the treatment of DM, there is an increased demand by patients to use natural products which have antidiabetic activity. Therefore, herbal drugs are gradually gaining popularity in the treatment of DM. The major qualities of herbal medicines are less costly, easily available, efficacious, and have low incidence of serious adverse effects.

In India, since time immemorial, patients with noninsulin dependent diabetes are treated orally with a variety of herbal drugs extracts. In Ayurveda literature, the numbers of plants were mentioned which have antidiabetic properties.

In view of this, the present study has investigated the effect of an ethanolic extract of leaves of Acacia catechu (A. catechu) in the management of DM in STZ-induced Wistar rats. STZ through its toxic effects induces oxidative stress in the $\beta$ cells of the pancreas, ${ }^{10}$ so it is frequently used to induce DM in experimental animals. The diabetogenic action of STZ is the direct result of irreversible damage to the pancreatic $\beta$ cells resulting in degranulation and loss of capacity to secrete insulin. ${ }^{11}$ The STZ effect on different organs has been extensively studied. Various studies have been done by using STZ to establish rat model of diabetic nephropathy. ${ }^{12}$

Acacia catechu wild belongs to Fabaceae family and mimosoideae subfamily. The generic name, "Acacia," comes from the Greek word akis, meaning a point ${ }^{13}$ which is distributed mainly in south India. Its bark root and heartwood has medicinal uses. The main chemical constituents of $A$. catechu are flavonoids, alkaloids, sugars, glycosides, and tannins. ${ }^{14}$ A. catechu wild has been shown to possess multifarious medicinal properties such as antibacterial, ${ }^{15}$ anticancer, ${ }^{16}$ hypoglycemic, antidiarrhoeal ${ }^{17,18}$ anti-inflammatory, antioxidant, ${ }^{19}$ hepatoprotective, ${ }^{20}$ sore throat, wound healing, etc.

However, systemic and scientific reports on the investigation of ethanolic extract of leaves of $A$. catechu for its effect on renal function are scarce. This study was designed to know the nephroprotective effects based on histopathological changes and antioxidant status in A. catechu with STZ-induced nephrotoxic rats.

\section{Materials and Methods}

\section{Plant Material}

The leaves of $A$. catechu were identified, collected, and authenticated by a Botanist. The leaves were dried in shade and powdered in our research laboratory with the help of pulverizer. The powder was subjected to soxhlet extraction with $95 \%$ ethyl alcohol for 72 hours at a temperature of 70 to $80^{\circ} \mathrm{C}$. The extract was concentrated to a small volume and then evaporated to dryness. This was then dissolved in sterile saline and administrated orally to the rats. Plant extract dose for experimental rats were selected based on in vivo acute toxicity study and its in vitro antioxidant potential compared with vitamin $\mathrm{C}$.

\section{Experimental Animals}

Male albino rats 9 to 11 weeks old, weighing between 200 and $250 \mathrm{~g}$ were used for the experiment. All animals were maintained under standard laboratory conditions, with a constant 12-hour light/dark cycle and controlled temperature $\left(25 \pm 2{ }^{\circ} \mathrm{C}\right)$ with free access to drinking water and pellet diet ad libitum.

This study was performed in a Committee for the Purpose of Control and Supervision of Experiments on Animals (CPCSEA) approved laboratory under registration number 115/1999/CPCSEA following all ethical practices as laid down in the guidelines for animal care. This study has been approved by the Institutional Animal Ethics Committee (IAEC; reference number KSHEMA/AEC/31/2011).

\section{Chemicals}

All the chemicals including STZ to induce DM and ether were purchased from Sri Durga Laboratory Equipment Supplies at Chilimbi main Road in Mangalore, citrate buffer (pH: 4.5) was used as a solvent to dissolve STZ.

\section{Induction of DM}

The animals were fasted for 16 to 18 hours with free access to water prior to the experiment. STZ of $45 \mathrm{mg} / \mathrm{kbw}$ was dissolved in $0.1 \mathrm{M}$ citrate buffer ( $\mathrm{pH} 4.5$ ) and the same was given a single dose of intraperitoneal administration (IP) to induce DM. ${ }^{21}$ Then $5 \%$ sucrose was supplemented for 24 hours to prevent the animals from fatal hypoglycemia. After 72 hours of STZ administration, fasting blood glucose (FBS) level using the glucometer from the tail vein was determined. The rats with an FBS more than $300 \mathrm{mg} / \mathrm{dL}$ were considered diabetic and included in the study.

\section{Methodology}

Male Wistar albino rats were selected based on their days of acclimatization. The rats were divided into three groups, namely, control (group A), STZ-induced diabetes mellitus (group B), and STZ-induced diabetes mellitus rats with A. catechu orally of $75 \mathrm{mg} / \mathrm{kg}$ body weight for 35 days, (group C) with each group having six rats $(n=6)$ weighing between 200 to $250 \mathrm{~g}$ each. They were kept fasting overnight (but with the free access to water). On the test day group $A$ received only water orally, group $B$ received a single dose of STZ at $45 \mathrm{mg} / \mathrm{kg}$ body weight IP, and group C received a single dose of STZ intraperitoneal and oral A. catechu for 35 days.

At the end of the 35th day of the observation period, the animals were deeply anesthetized with ether. All the animals were 
observed for any gross/macroscopic pathological changes, and the kidneys from the representative groups were removed and processed for the histological studies and tissue homogenate for antioxidants, GOT (glutamic oxaloacetic transaminase) and GPT (glutamic pyruvic transaminase).

\section{Preparation of Kidney Homogenate}

Kidneys were excised and cleaned with ice-cold saline and stored at $-20^{\circ} \mathrm{C}$ in the freezer. Tissues were thawed and homogenized in phosphate-buffered saline $\mathrm{pH}, 7.4$, centrifuged at $10,000 \mathrm{rpm}$ for 15 minutes using refrigerated centrifuge and supernatant was stored at $-20^{\circ} \mathrm{C}$. The supernatant was subjected to determination of GOT and GPT by Mohun and Cook method, ${ }^{22}$ SOD assay by Beauchamp and Fridovich method $^{23}$ and GSH-Px by Ellman's method. ${ }^{24}$

\section{Histopathological Examination}

Kidneys were kept in 10\% formalin for 48 hours (postfixation). By using standard histological procedures, paraffin blocks were prepared; sections were taken at $5 \mu$ thickness, stained with hematoxylin and eosin, and observations were done under the light microscope with $\times 40$ magnification for cytoarchitecture. ${ }^{25}$

\section{Statistical Analysis}

Statistical analysis is performed using Student's t-test and one-way analysis of variance (ANOVA) where ever it is applicable by SPSS, Version 22.0 software.

\section{Results}

SOD activity and renal GSH in group $\mathrm{C}$ is closer to group A when compared with group B where SOD and GSH activities were significantly elevated ( $p<0.05$; - Table1).

GOT and GPT in group C is closer to group A when compared with group B where GOT and GPT activities were significantly elevated ( $p<0.05$; - Table1).

Histological studies in group $C$ have revealed that the kidney were showing almost normal cytoarchitecture with group A (-Fig. 1). But in group B, rats' kidneys have shown moderate-to-severe degenerative features like dilated tubules, degenerated tubules, glomerular congestion, interstitial inflammatory infiltration, and atrophy of glomerulus seen with dilated glomerular space. The degenerated tubules cells with pyknotic nuclei and vacuolated cytoplasm. Sloughing of the epithelium was seen in tubular lumens (-Fig. 2). There was a significant change seen in the in tissue cytoarchitecture of group C (-Fig. 3) which clearly showed the antioxidant potency of the plant extract. This might be the major reason behind oxidative stress management in diabetic rats in this study. It shows that the plant extract has a nephroprotective effect which has favored the normal level of these biochemical parameters followed by the treatment.

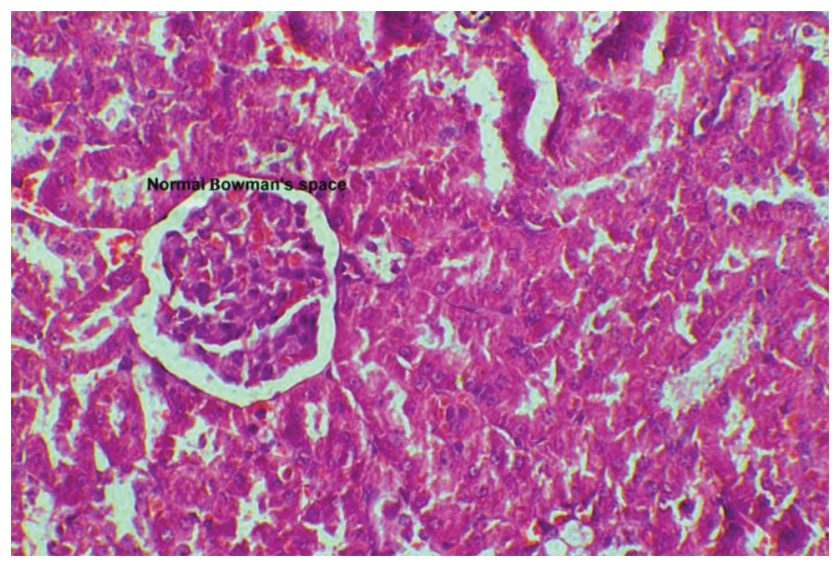

Fig. 1 A sectional representation of normal rat kidney (group A) at 40x magnification (hematoxylin and eosin stain) showing normal Glomeruli (G) with an intact Bowman's capsule, proximal convoluted tubules, and Distal convoluted tubules.

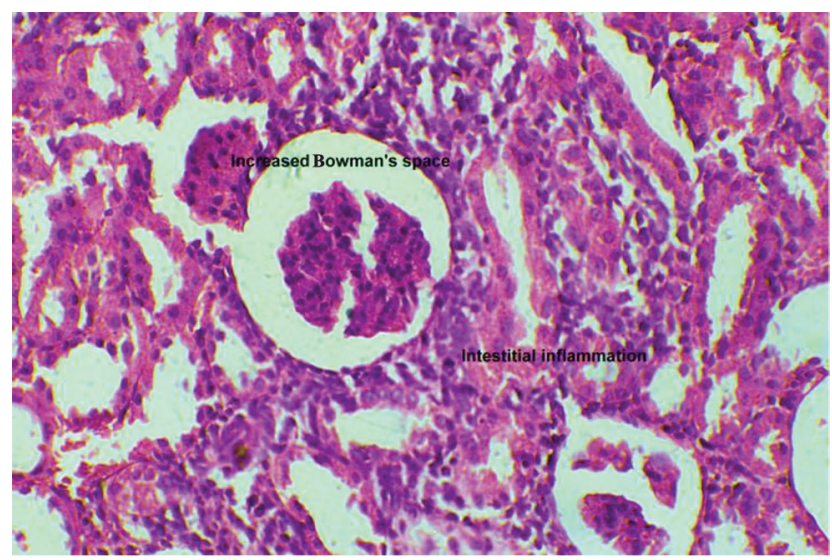

Fig. 2 A representative section of STZ diabetic control rat (Group B) kidney at 40x magnification (hematoxylin and eosin stain) showing increased Bowman's space, glomerular congestion, atrophy of glomerulus seen with dilated glomerular space and dilated tubules, degenerated tubules, interstitial inflammatory infiltration.

Table 1 The effect of Acacia catechu on functional enzymes in kidney tissues

\begin{tabular}{|l|l|l|l|l|}
\hline & SOD $(\mathbf{U} / \mathbf{g})$ & GSH $(\mu \mathrm{mole} / \mathrm{g})$ & GPT (U/g) & GOT (U/g) \\
\hline Normal & $312.32 \pm 10.13$ & $1.906 \pm 0.17$ & $0.523 \pm 0.0144$ & $1.613 \pm 0.025$ \\
\hline Diabetic control & $437.5 \pm 29.46^{\mathrm{a}}$ & $240.124 \pm 38.90^{\mathrm{a}}$ & $0.988 \pm 0.245^{\mathrm{a}}$ & $1.789 \pm 0.035$ \\
\hline $\begin{array}{l}\text { Diabetic + Acacia catechu } \\
\text { extract (75 mg/kg b.w.) }\end{array}$ & $337.41 \pm 70.18^{\mathrm{a}}$ & $1.994 \pm 0.55^{\mathrm{a}}$ & $0.686 \pm 0.063^{\mathrm{a}}$ & $1.643 \pm 0.169$ \\
\hline
\end{tabular}

Abbreviations: GOT, glutamic oxaloacetic transaminase; GPT, glutamic pyruvic transaminase; GSH, glutathione; SOD, superoxide dismutase. Note: Values are expressed as mean \pm standard deviation, $n=6$.

${ }^{a}$ Values are significantly different from the normal control group at $(p<0.05)$. 


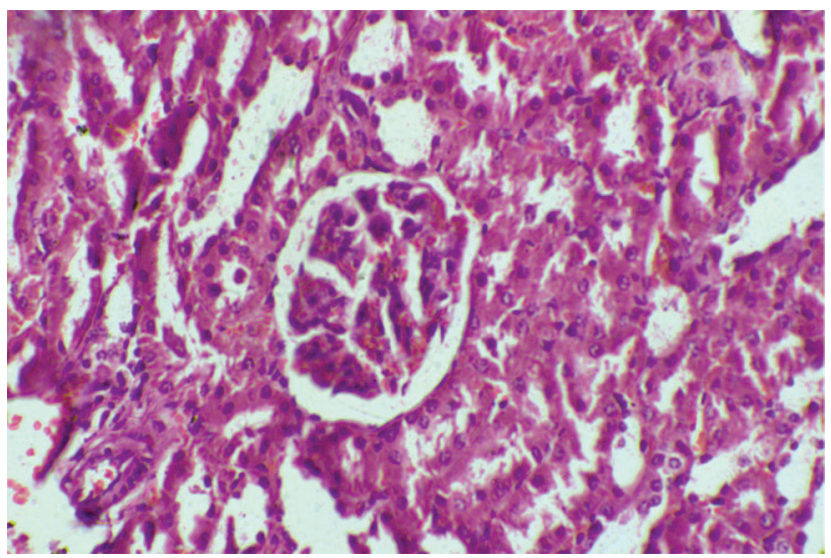

Fig. 3 A representative section of AC $(75 \mathrm{mg} / \mathrm{kg})$ extract treated group (Group C) kidney at 40x magnification (hematoxylin and eosin stain) showing sections with reduction in tubule dilation and degeneration with reduced Bowman's space, compared with STZ group. Glomerular congestion seen. There was no interstitial inflammatory infiltrate in any of the sections.

\section{Discussion}

The present work was aimed to study the antidiabetic activity of ethanolic extract of $A$. catechu leaves in STZ-induced diabetic rats. In our study, 72 hours after the injection of STZ to animals, significant hyperglycemia was observed in all rats. Hyperglycemia exerts its adverse effects by generation of ROS which then, in turn, can oxidize many other important biomolecules inducing "oxidative stress" (OS) ${ }^{26}$ and the increment of free radicals may lead to kidney cells damage, ${ }^{27}$ and the condition further deteriorates when the levels of antioxidants like GSH, GPx, SOD, vitamin C, etc., decrease which is usually observed in DM individuals, ${ }^{28}$ but some of the studies done had different observation, they had seen an increased levels, ${ }^{29,30}$ thus the role of antioxidants in DM is controversial.

On the contrary, the present investigation revealed an increase in the activity of antioxidant enzyme SOD and the concentration of GSH in the kidney tissue homogenates of STZ-induced DM. This may be because the diabetes was induced in healthy rats and the study period was very short; so, group B rats effectively increased the expression of antioxidant GSH and the enzyme SOD as a defense to reduce the oxidative stress developed by STZ in the kidney. The overexpression of these antioxidants might be an adaption response. SOD, for instance, is an important antidote to the toxic effect of superoxide anion is also increased due to the increasing dismutation of superoxide to hydrogen peroxide which is generally taken care of by the glutathione system. But in DM patients due to insulin deficiency, there is more of $\beta$ oxidation of fatty acids due to which hydrogen peroxide formation will increase. ${ }^{31}$ The higher free radical production and increased activity of antioxidant defense system during the early phase of the disease may be a phase of compensation which fails to sustain and hence leads to harmful effects of the unchecked oxidants causing complications of DM later on. In our study, after administration of A. catechu for 35 days to the STZ-induced DM rats, the antioxidant enzymes level reaches near to the normal level.

Evaluation of important housekeeping enzymes GOT and GPT activities in kidney tissues showed a significant increase in the activity in untreated STZ-induced DM rats compared with healthy group A ( - Table 1 ). While the activity of these enzymes reduced significantly in group $C$ in comparison to group A. These alterations in the activity of mentioned enzymes in untreated DM rats may be due to the metabolic abnormalities or cellular injuries. ${ }^{32}$ It has been reported that the increase in GOT and GPT activities in the kidney tissues of STZ treated rats is due to the subtle membrane changes that allow to the passage of intracellular enzymes to the extracellular space. ${ }^{33}$

Our data have shown that activity of the above-mentioned enzymes in the STZ-induced DM was nearly normalized by the A. catechu.

In accordance with the results obtained in biochemical analysis, the histological analysis in DM rats supplemented with extract of $A$. catechu showed reduction in tubule dilation and degeneration with normal glomerular space compared with STZ group. Glomerular congestion was also reduced. There was no interstitial inflammatory infiltrate in any of the sections compared with STZ treated group. By virtue of its antioxidant property, A. catechu extract was able to render nephroprotection in these models by attenuating oxidative stress. So, it was speculated that the nephroprotective effect of $A$. catechu might be due to its antioxidant property. The plant extract might contain bioactive components that have the potential to reverse the undesirable changes in the kidney associated with hyperglycemia-induced oxidative stress. ${ }^{34}$ Thus, a corrective measure even on the histology of the kidney was noticed.

\section{Conclusion}

A. catechu was evaluated for its anti-diabetic property in STZ-induced DM rats for 35 days at the dose of $75 \mathrm{mg} / \mathrm{kg}$ body weight. The antioxidants levels GOT and GPT and histological studies have suggested that the A. catechu have some active principals which are antidiabetic and nephroprotective. Further studies are to isolate the active components of A. catechu which is needed for the treatment of DM.

\section{Authors' Contributions}

P.D. designed and worked on the experiment, R.H. collected the review of the literature and also planned for the experiment, and G.S. helped in planning and statistical analysis of the work. All three authors equally contributed to the overall study.

\section{Conflict of Interest}

None declared.

\section{Acknowledgment}

The authors would like to thank the Management of K.S. Hegde Medical Academy at Deralakatte in Mangalore, Karnataka. 


\section{References}

1 Whiting DR, Guariguata L, Weil C, Shaw J. IDF diabetes atlas: global estimates of the prevalence of diabetes for 2011 and 2030. Diabetes Res Clin Pract 2011;94(3):311-321

2 Ritz E, Orth SR. Nephropathy in patients with type 2 diabetes mellitus. N Engl J Med 1999;341(15):1127-1133

3 Matough FA, Budin SB, Hamid ZA, Alwahaibi N, Mohamed J. The role of oxidative stress and antioxidants in diabetic complications. Sultan Qaboos Univ Med J 2012;12(1):5-18

4 Je HD, Shin CY, Park HS, Huh IH, Sohn UD. The comparison of vitamin $C$ and vitamin $\mathrm{E}$ on the protein oxidation of diabetic rats. J Auton Pharmacol 2001;21(5-6):231-236

5 Yildirim 0 , Büyükbingöl $Z$. In vivo effect of vitamin $C$ with cobalt on oxidative stress in experimental diabetic rat kidney. Diabetes Nutr Metab 2003;16(4):208-213

6 Savu O, Ionescu-Tirgoviste C, Atanasiu V, Gaman L, Papacocea $\mathrm{R}$, Stoian I. Increase in total antioxidant capacity of plasma despite high levels of oxidative stress in uncomplicated type 2 diabetes mellitus. J Int Med Res 2012;40(2):709-716

7 Viswanathan V. Prevention of Diabetic Nephropathy: A diabetologist's perspective. Indian J Nephrol 2004;14:157-162

8 Rosario RF, Prabhakar S. Lipids and diabetic nephropathy. Curr Diab Rep 2006;6(6):455-462

9 Venkatesh S, Reddy GD, Reddy BM, Ramesh M, Rao AV. Antihyperglycemic activity of Caralluma attenuata. Fitoterapia 2003;74(3):274-279

10 Deeds MC, Anderson JM, Armstrong AS, et al. Single dose streptozotocin-induced diabetes: considerations for study design in islet transplantation models. Lab Anim 2011;45(3):131-140

11 GU D, Arnush M, Sarvetnic N. Endocrine/exocrine intermediate cells in STZ treated Ins-IFNgamma transgenic mice. Pancreas $1997 ; 15(3): 246-250$

12 Tesch GH, Allen TJ. Rodent models of streptozotocininduced diabetic nephropathy. Nephrology (Carlton) 2007;12(3):261-266

13 Lakshmi T, Anitha R, Geetha RV. Acacia catechu willd - A gift from ayurveda to mankind - A Review. T. Ph. Res. 2011;5(2):273-293

14 Monu MP, Kadian R, Sharma K. Phytopharmacology of acacia catechu willd: a review. World J Pharm Sci 2014;3 (11):1380-1389

15 Pfaller W, Gstraunthaler G. Nephrotoxicity testing in vitrowhat we know and what we need to know. Environ Health Perspect 1998;106(2):559-569

16 Nadumane KV, Nair S. Evaluation of the anticancer and cytotoxic potentials of Acacia catechu extracts in vitro. J Nat Pharmaceuticals. 2011;2(4):190-195

17 Ray D, Sharatchandra K, Thokchom I. Antipyretic, antidiarrhoeal, hypoglycaemic and hepatoprotective activities of ethyl acetate extract of Acacia catechu Wild in albino rats. Ind J Pharmacol 2006;38(6):408-413

18 Gayathri DV, Lanitha J, Devi R, Sreekala, Prabhakaran VA. Pharmacognostical studies on Acacia catechu willd and identification of antioxidant principles. Int J Pharm Sci 2011;3(2):108-111

19 Guleria S, Tiku AK, Singh G, Vyas D, Bhardwaj A. Antioxidant activity and protective effect against plasmid DNA strand scission of leaf, bark, and heartwood extracts from Acacia catechu. J Food Sci 2011;76(7):C959-C964

20 Pingale SS. Heptoprotection by Acacia catechu in CCL4 Induced Liver Dysfunction. Inter J of Pharmaceutical Sciences Rev. and Res. 2010;5(1):150-154

21 Nagarchi K, Ahmed S, Sabus A, Saheb SH. Effect of STZ on Glucose levels in Albino Wister Rats J. Pharm. Sci. \& Res. 2015;7(2):67-69

22 Mohun(A.). Cook(P.). Simple methods for measuring serum levels of the glutamate-oxaloacetate and glutamic pyruvic transaminases in routine laboratory. J Clin Puth 1957;10:394-399

23 Beauchamp C, Fridovich I. Superoxide dismutase: improved assays and an assay applicable to acrylamide gels. Anal Biochem 1971;44(1):276-287

24 Ellman GL. Tissue sulfhydryl groups. Arch Biochem Biophys 1959;82(1):70-77

25 Teoh SL, Abd Latiff A, Das S. Histological changes in the kidneys of experimental diabetic rats fed with Momordica charantia (bitter gourd) extract. Rom J Morphol Embryol 2010;51(1):91-95

26 Haneda M. [Mechanisms for the development and progression of diabetic nephropathy]. Nihon Rinsho 2006;64(Suppl 2): 427-432

27 Sharma S, Kulkarni SK, Chopra K. Curcumin, the active principle of turmeric (Curcuma longa), ameliorates diabetic nephropathy in rats. Clin Exp Pharmacol Physiol 2006;33(10):940-945

28 Mansouri E, Panahi M, Ghaffari MA, Ghorbani A. Effects of grape seed proanthocyanidin extract on oxidative stress induced by diabetes in rat kidney. Iran Biomed J 2011;15(3):100-106

29 Uzel N, Sivas A, Uysal M, Oz H. Erythrocyte lipid peroxidation and glutathione peroxidase activities in patients with diabetes mellitus. Horm Metab Res 1987;19(2):89-90

30 Pasaoglu H, Sancak B, Bukan N. Lipid peroxidation and resistance to oxidation in patients with type 2 diabetes mellitus. Tohoku J Exp Med 2004;203(3):211-218

31 Sandhya Pillai Nair NC. Shah, R.M. Shah. Alteration in enzymatic antioxidant defense in diabetes mellitus. Biomed Res 2012;23(3):402-404

32 Baghshani H, Ghodsi V. Evaluation of some enzymatic changes in the liver and kidney of rats following exposure to sub-lethal concentration of potassium cyanide. Iranian Journal of Toxicology. 2016;10(4):9-12

33 Zafar M, et al. Altered kidney morphology and enzymes in STZ induced diabetic rats. Int J Morphol 2009;27(3):783-790

34 Saha MR, Dey P, Begum S, et al. Effect of Acacia catechu (L.f.) Willd. on oxidative stress with possible implications in Alleviating Selected Cognitive Disorders. PLoS One 2016;11(3):e0150574 